\title{
MHD-DRIVEN KINETIC DISSIPATION IN THE SOLAR WIND AND CORONA
}

\author{
R. J. Leamon, W. H. Matthaeus, C. W. Smith, G. P. Zank, and D. J. Mullan \\ Bartol Research Institute, University of Delaware, Newark, DE 19716.; leamon@bartol.udel.edu, yswhm@bartol.udel.edu, \\ chuck@bartol.udel.edu, zank@bartol.udel.edu, mullan@bartol.udel.edu \\ AND \\ S. OUGHTON \\ Department of Mathematics, University College London, London WC1E 6BT, UK; sean@math.ucl.ac.uk \\ Received 1999 October 7; accepted 2000 February 17
}

\begin{abstract}
Mechanisms for the deposition of heat in the lower coronal plasma are discussed, emphasizing recent attempts to reconcile the fluid and kinetic perspectives. Structures at magnetohydrodynamic (MHD) scales may drive a nonlinear cascade, preferentially exciting high perpendicular wavenumber fluctuations. Relevant dissipative kinetic processes must be identified that can absorb the associated energy flux. The relationship between the MHD cascade and direct cyclotron absorption, including cyclotron sweep, is discussed. We conclude that for coronal and solar wind parameters the perpendicular cascade cannot be neglected and may be more rapid than cyclotron sweep. Solar wind observational evidence suggests the relevance of the ion inertial scale, which is associated with current sheet thickness during reconnection. We conclude that a significant fraction of dissipation in the corona and solar wind likely proceeds through a perpendicular cascade and small-scale reconnection, coupled to kinetic processes that act at oblique wavevectors.
\end{abstract}

Subject headings: MHD - solar wind - Sun: corona - Sun: magnetic fields - turbulence

\section{INTRODUCTION}

A recurring theme in recent studies of the physics of the corona has been to identify the mechanism by which heat is deposited within two or three solar radii of the photosphere in sufficient quantities to both accelerate the solar wind (Holzer 1977; Habbal et al. 1995; Kohl et al. 1995; Grall et al. 1996; Cuseri et al. 1999) and to account for high ion temperatures inferred from recent $\mathrm{SOHO}$ observations (Kohl et al. 1997; Cranmer, Field, \& Kohl 1999). Similarly, heating in the extended solar wind is strongly suggested by the observed nonadiabatic radial temperature profile (Richardson et al. 1995). Magnetic fluctuations, including waves and turbulence, are a likely source of energy to account for this heating, both in the lower corona, where substantial dissipation is implied at distances less than 2-3 $R_{\odot}$ (McKenzie, Banaszkiewicz, Axford 1995), and in the solar wind where significant heating occurs to at least 50 AU (Richardson et al. 1995; Zank, Matthaeus, \& Smith 1996). Of the models proposed to explain these observational constraints, the Axford \& McKenzie (1997; see also McKenzie et al. 1995) model is representative of those that rely upon the direct cyclotron resonant absorption of relatively high-frequency $(\sim \mathrm{kHz})$ waves. Such models provide natural explanations for the high perpendicular temperatures of both the protons and the minor ions. At another extreme, models based upon MHD cascade involve direct participation of broader band, lower frequency fluctuations (Hollweg 1986; Hollweg \& Johnson 1988; Matthaeus et al. 1999b). In the cascade approach nonlinear wave-wave couplings cause energy transfer across a wide range of spatial scales, leading to efficient small-scale kinetic dissipation. Cascade models have been employed successfully to model solar wind observations $(\mathrm{Tu}, \mathrm{Pu}, \&$ Wei 1984; Matthaeus et al. 1999b). (Other models have been proposed that involve damping of low-frequency modes by other mechanisms, such as noncyclotron-resonant absorp- tion [Cuseri et al. 1999] or mode conversion [Kaghashvili 1999]).

Cyclotron absorption and cascade may contribute concurrently to heating. However, to date there has been relatively little progress in understanding the detailed relationship between the associated fluid and kinetic scale processes in collisionless plasmas. The subject of the present paper is the interplay between the spectral cascade and kinetic damping - a complex issue that is fundamental to the heating of many astrophysical plasmas. In the following sections we discuss physical and observational issues of relevance to direct cyclotron absorption models, as well as alternatives that involve MHD cascade as an essential feature.

In $\S 2$ we discuss the relationship between cascade and small-scale dissipation, emphasizing the role of conservative MHD spectral transfer and anisotropic MHD couplings. We obtain estimates for the relative importance of the cascade and cyclotron sweep mechanisms. In $\S 3$ we summarize recent (observational) solar wind results relevant to anisotropy and heating by perpendicular cascade. We describe some new results on spectral steepening (e.g., Leamon et al. 1998b; Goldstein et al. 1994), on the basis of which we suggest that the solar wind dissipation range may have its onset at the ion inertial scale, and at an orientation highly oblique to the mean field. In $\S 4$ we summarize the perspective that solar wind and coronal dissipation may involve significant contributions from quasi-twodimensional (quasi-2D) reconnection, and kinetic processes associated with oblique current sheet formation, with a (dominant) perpendicular cascade channeling the energy to the associated wavevectors.

\section{DISSIPATION OF MHD FLUCTUATIONS}

It is useful to consider formally how the MHD fluid scale energy $E$ changes dynamically in a Fourier representation. 
In this context, by energy we mean the macroscopic fluid scale energy. This includes contributions from the kinetic energy of the velocity fluctuations and from the magnetic fluctuations; however, the thermal energy is not included in $E$. Dissipation converts fluid energy $E$ into thermal energy. A useful physical picture associated with a turbulent energy cascade is that of an energy "pipeline," which transfers energy through intermediate steps from the large, energycontaining scales to the small-scales where direct dissipation is effective. Formally, we can use a wavevector decomposition of the energy $E=\int E(\boldsymbol{k}) d^{3} k$, to write an equation for the time variation of the energy density at wavevector $\boldsymbol{k}$, which includes the effects of spectral transfer $T$, dissipation $D$, and sources $S$ :

$$
\frac{\partial E(\boldsymbol{k}, t)}{\partial t}=T(\boldsymbol{k}, t)+D(\boldsymbol{k}, t)+S(\boldsymbol{k}, t) .
$$

The quantity $T(\boldsymbol{k})$ is the Fourier transform of the nonlinear terms in the MHD equations that are responsible for spectral transfer. Loosely speaking, $T(\boldsymbol{k})$ is the pipeline referred to above. It is related to the energy transfer function in isotropic turbulence (Panchev 1971) and represents transfer of energy from all wavevectors into excitations near wavevector $\boldsymbol{k}$.

The dissipation function $D(k)$ may be of the form

$$
D(\boldsymbol{k})=[\partial E(\boldsymbol{k}) / \partial t]_{\mathrm{diss}}=-\gamma(\boldsymbol{k}) E(\boldsymbol{k}) .
$$

This form is standard for hydrodynamic dissipation with viscosity $v$, in which case $\gamma(\boldsymbol{k}) \rightarrow v k^{2}$. In a space physics application, however, it is more likely that $\gamma(\boldsymbol{k})$ might be approximated as a linear damping rate computed from linearized Vlasov theory (Leamon et al. 1999). (For simplicity we will discuss only the total MHD fluctuation energy $E$, rather than the kinetic and magnetic energy separately.) Energy sources at wavevector $\boldsymbol{k}$, designated by $S(\boldsymbol{k})$, supply energy at a total rate $S$, and may be associated with largescale shear, injection by wave-particle interactions, etc. Spectral transfer is conservative $\left(\int T(\boldsymbol{k}) d^{3} k=0\right)$, and thus the time rate of change of the total energy $E$ is the sum of sources and dissipation,

$$
\frac{\partial E}{\partial t}=S-\int \gamma(\boldsymbol{k}) E(\boldsymbol{k}) d^{3} k,
$$

where the integral extends over all wavevectors.

Clearly, $\gamma(\boldsymbol{k})$ does not alone determine the dissipation rate. $E(\boldsymbol{k})$ is also needed, and this depends upon spectral transfer through $T(\boldsymbol{k})$ in equation (1). This is, in essence, the central problem of turbulence (see, e.g., Lesieur 1990). Whatever the strategy might be for dealing with $T(\boldsymbol{k})$, one cannot evaluate the rate of dissipation of energy without knowledge of both $\gamma(\boldsymbol{k})$ and $E(\boldsymbol{k})$. Thus, we need to simultaneously confront the issues of MHD spectral transfer (governed by large-scale dynamics) and kinetic dissipation mechanisms (controlled by microphysics).

\subsection{Cascade, Sweep, and Spectral Replenishment}

To illustrate the consistency of a full cascade model, assume for the moment a simplified situation wherein all the dissipation occurs at large wavenumbers $k>k_{0}$. Thus, fluctuations within a wavenumber-space sphere of radius $k_{0}$ obey dynamics that are, in effect, ideal and dissipationless. In practice, for a low-beta plasma, and a spectrum of Alfvén waves, $k_{0}$ would probably be chosen to be some fractional multiple of $2 \pi / \rho_{\mathrm{ii}}$, where $\rho_{\mathrm{ii}}=c / \omega_{\mathrm{pi}}$ is the ion inertial length, $c$ the speed of light and $\omega_{\mathrm{pi}}$ the plasma frequency. It may be convenient to think of $k_{0}$ as a wavenumber in the inertial range. For now, assume that $\gamma(\boldsymbol{k})=0$ when $k<k_{0}$. Thus, integrating equation (1), over the large-scale (“energy-containing") eddies, we obtain

$$
\frac{d E\left(k<k_{0}\right)}{d t}=S_{0}(t)-F_{0}\left(k_{0}, t\right) .
$$

Here $S_{0}$ is the net rate of energy supplied by forcing (assumed here to be at the larger scales $k<k_{0}$ ). The second term on the right hand side of equation (4) denotes the energy per unit time transferred from wavenumbers lower than $k_{0}$ to higher wavenumbers. Flux functions of this type can be defined more formally (see the Appendix).

The evolution equation for small-scale energy is

$$
\frac{d E\left(k>k_{0}\right)}{d t}=F_{0}\left(k_{0}, t\right)-\int \gamma(\boldsymbol{k}) E(\boldsymbol{k}, t) d^{3} k,
$$

obtained by integrating over $k>k_{0}$, or by using equation (3). For strong turbulence the energy flux is usually controlled by the self-interactions of the larger scale eddies. So, for example, in hydrodynamics, or zero cross helicity MHD (see, e.g., Hossain et al. 1995), the energy transfer rate becomes nearly independent of $k$ for a range of $k$ (the inertial range). For $k=k_{0}$ within this range $F_{0}\left(k_{0}\right)=\epsilon \approx u^{3} / \lambda$. Here $u$ is the rms velocity fluctuation and $\lambda$ is a characteristic energy-containing length scale. For steady driven turbulence the total fluctuation level adjusts so that $F_{0} \approx S_{0}$. While large-scale eddies control the total decay rate, spectral transfer determines how the energy is dissipated at small scales. We now discuss two possible approaches to studying spectral transfer and dissipation.

The first is the limit of weak or vanishing spectral transfer $(T \approx 0)$, which implies no spectral replenishment. An important example of this is the "cyclotron sweep mechanism," in which the decay rates depend on the local proton cyclotron frequency $\Omega_{\mathrm{ci}}$ (Hollweg \& Turner 1978; Schwartz, Feldman, \& Gary 1981; Tu \& Marsch 1997), and turbulence is transported through regions in which $\Omega_{\mathrm{ci}}$ varies. For example, in the solar wind and corona $\Omega_{\mathrm{ci}}$ decreases with increasing radial distance $R$. As a result, outward moving fluctuations are transported into regions where the damping occurs at progressively lower frequencies. The dissipation absorbs the energy available at the local gyrofrequency, and this process "sweeps" through the spectrum toward lower frequencies (and lower wavenumbers), leaving in its wake a range of energy-depleted frequencies. Further discussion of this mechanism is given in the next section.

A second and very different approximation is obtained by assuming that the energy spectrum is known (Isenberg \& Hollweg 1983; Leamon et al. 1999). (Often a simple functional form such as a power law is employed.) One might justify this by assuming that spectral transfer is strong enough to replenish losses due to dissipation, so that the spectrum is maintained at a certain level. This is exact in steady-state. Observations can be invoked to defend a particular functional form. Provided that the full threedimensional energy spectrum is specified correctly, the transfer function is then irrelevant.

\subsection{Anisotropic MHD:}

Wavenumber and Frequency Cascades

There are conditions that must be satisfied to arrive at a well-posed model of spectral transfer and dissipation. For 
example, as we mentioned above, steady spectral replenishment implies a concomitant transfer function to maintain the spectrum. The only completely reliable way to compute the transfer rates into the dissipation range is to solve the full nonlinear problem for all dynamically important spatial scales. Unfortunately, this is seldom feasible. Thus, it is important to construct models that conform to the known properties of spectral transfer. This requirement seems to have not been fully appreciated previously.

There is considerable evidence that turbulent MHD spectral transfer in the presence of a mean magnetic field $\left(\boldsymbol{B}_{0}\right)$ is anisotropic, favoring production of structures with small transverse scales (relative to $\boldsymbol{B}_{0}$ ). Descriptions of this robust form of dynamically generated anisotropy come from theory (Montgomery \& Turner 1981; Montgomery 1982; Zank \& Matthaeus 1992, 1993; Kinney \& McWilliams 1998) and from simulations (Shebalin, Matthaeus, \& Montgomery 1983; Oughton, Priest, \& Matthaeus 1994b; Matthaeus et al. 1998). In the corona, two important parameters - the plasma beta $\left(\beta_{\mathrm{p}}\right)$ and $b / B_{0}(b$ the rms magnetic fluctuation) - are expected to be small. This situation favors the production of "quasi-2D" fluctuations, i.e., those with wavevectors $\boldsymbol{k}$ such that $\boldsymbol{k} \cdot \boldsymbol{B}_{0} \approx 0$, produced by spectral transfer that vigorously moves energy toward higher transverse wavenumbers.

The anisotropy of the cascade can have a significant impact in determining which kinetic processes are relevant to the deposition of heat. In steady-state, the amount of energy dissipated per unit time at high parallel wavenumbers must match the rate of supply by the parallel cascade. However, the rate of energy supply to parallel wavenumbers can be quite limited, and thus the amount of dissipation at high parallel wavenumbers may also be constrained.

The physical basis for the above remarks is clearly set out in the references; here we make the central point quantitatively. We employ a three-dimensional incompressible MHD spectral method simulation and the same approach as used by Matthaeus et al. (1998). The isotropic dissipation involves equal scalar dissipation coefficients, the resistivity $\mu$ and the viscosity $v$. This type of simulation was used previously to investigate the dynamical emergence of spectral anisotropy from isotropic initial states. Here we illustrate a closely related issue, namely the relative contributions to dissipation due to parallel gradients and to perpendicular gradients. The former quantity is $D_{\|} \propto v \bar{k}_{\|}^{2}$, while the latter is $D_{\perp} \propto \nu \bar{k}_{\perp}^{2}$, where $\bar{k}_{\|}$and $\bar{k}_{\perp}$ are mean wavenumbers defined in the parallel and perpendicular directions, respectively. In a steady state, the two contributions sum to the total energy decay rate $\epsilon=\epsilon_{\|}+\epsilon_{\perp}=$ $v \sum k^{2} E_{v}(\boldsymbol{k})+\mu \sum k^{2} E_{b}(\boldsymbol{k})$, where $E_{v}(\boldsymbol{k})$ and $E_{b}(\boldsymbol{k})$ denote the energy spectra for the velocity and magnetic field fluctuations, respectively.

In a near steady-state, the dissipation in each part of wavevector space must be balanced by spectral transfer. For example, parallel dissipation $\epsilon_{\|}$must be balanced by spectral transfer in the parallel direction. This can be quantified in terms of the spectral flux functions $F_{\perp}$ and $F_{\|}$that measure spectral transfer in the perpendicular and parallel directions, just as $F_{0}$ in the previous section is a measure of the net spectral flux to higher wavenumbers. The Appendix provides more formal definitions. In a near steady state, we expect that $F_{\|} \approx \epsilon_{\|}$and $F_{\perp} \approx \epsilon_{\perp}$. In this way, one may study the anisotropy of spectral transfer by examining the anisotropy of the dissipation.
The two cases shown in Figure 1 have identical parameters, with one exception - the value of the uniform magnetic field strength, taken as either $B_{0}=0$ or $B_{0}=8$ (in units where the initial rms fluctuation is unity). It is evident that when the mean magnetic field is strong, dissipation is dominated by the contribution from perpendicular wavevectors. By contrast, when $B_{0}=0$, dissipation due to perpendicular and parallel contributions is almost equal (on a per component basis).

For the illustrated case, $\gamma(\boldsymbol{k})=v k^{2}$ is isotropic. Thus, the disparity in dissipation rates is due to anisotropic spectral transfer. The stronger perpendicular cascade, which is effective at inertial range scales and smaller, leads to unequal dissipation, $\epsilon_{\perp}>\epsilon_{\|}$in the simulation. The fact that this condition is maintained implies that the parallel cascade is weak and that therefore $F_{\perp}>F_{\|}$.

This effect has been seen in compressible and incompressible MHD simulations, and in both decaying and driven situations (Matthaeus et al. 1998). Thus, in the corona, where $\beta_{\mathrm{p}} \ll 1$ and $b / B_{0} \ll 1$, we can expect the parallel cascade to be slow-perhaps even negligible - in comparison to the perpendicular cascade.

A revealing corollary is that the frequency cascade (defined presently) may be very much slower than the wavenumber cascade. For low $\beta_{\mathrm{p}}$ let us consider a nearly incompressible MHD fluid (see, e.g., Zank \& Matthaeus 1992, 1993), in which case the dominant wave mode is the Alfvén wave, and the wave frequency is given by $2 \pi f=k_{\|} V_{\mathrm{A}}$,
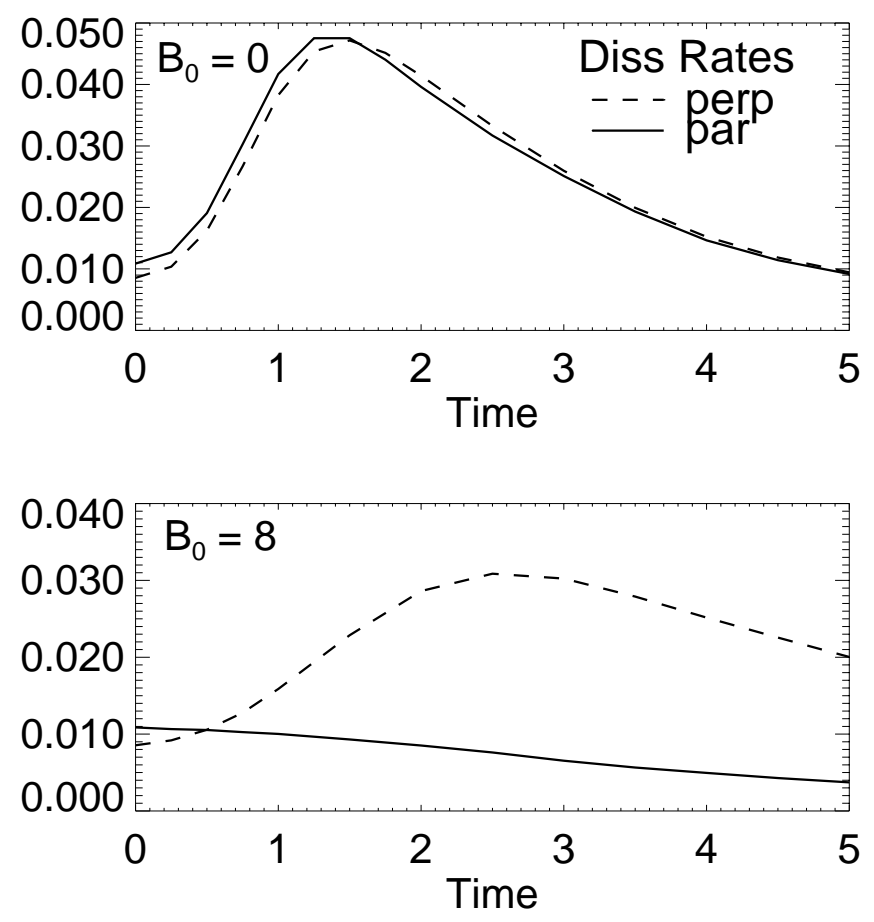

FIG. 1.-Contributions to the total dissipation rate from parallel and perpendicular gradients (per component). The data is taken from $64^{3}$ spectral method incompressible MHD simulations at equal mechanical and magnetic Reynolds numbers $R=200$. Simulations shown are identical decaying initial value problems, with unit initial fluctuation energy density, differing only in the value of the uniform constant mean magnetic field strength: $B_{0}=0$ (top) and $B_{0}=8$ (bottom). Parallel and perpendicular contributions are very nearly equal in the $B_{0}=0$ case, which remains close to isotropic. The strong mean-field case develops similar level of perpendicular dissipation, but parallel dissipation is reduced because of the suppression of parallel spectral transfer. The dissipation function in these cases is isotropic, $\gamma(\boldsymbol{k})=v k^{2}$. 
where $V_{\mathrm{A}}$ is the Alfven speed. Consequently the cascade in wave frequency is identical to the parallel portion of the wavenumber cascade. However, the wavenumber cascade is dominated by the perpendicular cascade, so that the wave frequency cascade is not a good measure of the overall cascade rate. In particular, strong spectral transfer does not imply a concomitant strong cascade in wave frequency. In steady-state the flux of energy into high-frequency modes may be only a small fraction of the total energy flux to high wavenumbers.

\subsection{Cascade and Sweep Rates}

Parallel cascade is expected to be suppressed relative to the perpendicular cascade, but a cyclotron sweep mechanism can still account for substantial dissipation of highfrequency Alfvén waves (Schwartz et al. 1981; Tu \& Marsch 1997). It is evidently of interest to compare the expected rates of dissipation due to cyclotron sweep and perpendicular cascade dissipation mechanisms.

Let us denote the perpendicular cascade heating rate as $\epsilon_{\perp}$ and the cyclotron sweep heating rate as $\epsilon_{\mathrm{cyc}}$. The former is estimated in the standard way (Matthaeus et al. 1999b) as $\epsilon_{\perp}=u^{3} / \lambda_{\perp}$ where $u$ is the rms turbulent velocity and $\lambda_{\perp}$ is the similarity scale, or energy-containing scale of the quasi2D fluctuations, often taken to be the perpendicular correlation length. We assume this kind of perpendicular cascade is relevant to the coronal plasma.

The cyclotron sweep damping rate may be estimated as $\epsilon_{\text {cyc }} \sim\left(V_{\mathrm{SW}}+V_{\mathrm{A}}\right) P\left(f_{\mathrm{ci}}\right) \mathrm{d} f_{\mathrm{ci}} / \mathrm{d} R$ where $V_{\mathrm{Sw}}$ is the flow speed, $V_{\mathrm{A}}$ the Alfvén speed, $P(f)$ the frequency dependent power spectrum of the fluctuations, and $f_{\mathrm{ci}}$ is the local proton gyrofrequency, varying with heliocentric radius $R$ (Tu \& Marsch 1997; Schwartz et al. 1981). Defining $\lambda_{h}$ as the scale height for gyrofrequency variation, and $u_{\text {diss }}^{2}$ to be the energy in dissipation range fluctuations (Leamon et al. $1998 \mathrm{~b})$ we find that $\epsilon_{\mathrm{cyc}} \sim\left(V_{\mathrm{SW}}+V_{\mathrm{A}}\right) u_{\mathrm{diss}}^{2} / \lambda_{h}$. Regrouping terms, (and specializing to the corona in which $V_{\mathrm{A}} \gg V_{\mathrm{SW}}$ ) we estimate that the ratio of cascade heating rate to gyrofrequency sweep heating rate is

$$
\frac{\epsilon_{\perp}}{\epsilon_{\mathrm{cyc}}} \approx\left(\frac{u^{2}}{u_{\mathrm{diss}}^{2}}\right)\left(\frac{u}{V_{\mathrm{A}}}\right)\left(\frac{\lambda_{h}}{\lambda_{\perp}}\right) .
$$

For each of these factors we will form a high estimate and a low estimate.

The first of these factors expresses essentially the ratio of fluctuation energy at the correlation length scale $\lambda_{\perp}$ to that at the dissipation scale $\lambda_{\text {diss }}$. Assuming that a single powerlaw $k_{\perp}^{-5 / 3}$ spectrum is valid from the energy containing scales down to the dissipative scales, this factor is of order $\left(\lambda_{\perp} / \lambda_{\text {diss }}\right)^{2 / 3}$. Let us estimate the perpendicular energy containing scale as $\lambda_{\perp} \approx 3 \times 10^{4} \mathrm{~km}$, the typical supergranulation scale. Below we shall argue that $\lambda_{\text {diss }} \approx \rho_{i i}$, the ion inertial scale where $\rho_{i i}=c / \omega_{\mathrm{pi}}=V_{\mathrm{A}} / \Omega_{c i}=2.3 \times 10^{2}$ $\mathrm{km} / \sqrt{n}$ and $n$ is the proton number density in $\mathrm{cm}^{-3}$. For a low coronal number density of $n \sim 2 \times 10^{7} \mathrm{~cm}^{-3}$, we have $\lambda_{\text {diss }} \approx 5 \times 10^{-2} \mathrm{~km}$. Thus, for a $k^{-5 / 3}$ inertial range, our first factor may be estimated as $\left(3 \times 10^{4} /\right.$ $\left.5 \times 10^{-2}\right)^{2 / 3} \approx 7.1 \times 10^{3}$. This is our high estimate. A low estimate may be obtained by allowing the spectrum to be very flat, say $1 / f$ as assumed by Tu \& Marsch (1997). In this case our first factor is $\approx \ln \left(3 \times 10^{4} / 5 \times 10^{-2}\right) \approx 13$.

To estimate the second factor, we note that turbulent velocities in the solar wind reach values of $u \approx 200-300 \mathrm{~km}$ $\mathrm{s}^{-1}$ (comparable to the mean flow speed) at radial distances of order $10 R_{\odot}$ (Scott, Coles, \& Bourgois 1983). At these distances the flow speed may be comparable to the Alfvén speed (Foukal 1990), in which case the second term in equation (6) is of order unity. Closer to the sun, $u$ decreases (Scott et al. 1983) while $V_{\mathrm{A}}$ is expected to increase. As a result, $u / V_{\mathrm{A}}$ is expected to be less than unity in the corona. On the basis of UVCS/SOHO observations of line broadening, Cranmer et al (1999) suggest total random motions of up to $200 \mathrm{~km} \mathrm{~s}^{-1}$ at $r \sim 2 R_{\odot}$, most of which may be thermal. There is some evidence from SUMER observations (Chae, Schühle, \& Lemaire 1998) at somewhat lower altitudes $\left(r \leq 1.05 R_{\odot}\right)$ and from EUV line broadening (Hassler et al. 1990) $\left(r \approx 1.1 R_{\odot}\right)$ that turbulent motions up to several tens of $\mathrm{km} \mathrm{s}^{-1}$ may be present. In spite of these suggestions, we emphasize that currently available observations do not provide unambiguous estimates of the coronal value of $u / V_{\mathrm{A}}$. At present we shall assume that $u=30 \mathrm{~km} \mathrm{~s}^{-1}$, which is not unreasonable in view of the available observations. This is also consistent with the boundary conditions assumed by Tu \& Marsch (1997). To form a low estimate of the factor $u / V_{\mathrm{A}}$ we adopt a "fast" Alfvén speed of $1000 \mathrm{~km} \mathrm{~s}^{-1}$, so that $u / V_{\mathrm{A}} \approx 1 / 33$. For a "high" estimate, $V_{\mathrm{A}}=200 \mathrm{~km} \mathrm{~s}^{-1}$ provides that $u / V_{\mathrm{A}} \approx 1 / 7$.

The final factor shares with the first factor the difficulty that the transverse correlation scale of coronal fluctuations is unknown. Based upon remote sensing of anisotropic density fluctuations (Grall et al. 1997), we might expect that $\lambda_{\perp} \ll R_{\odot}$. However, it is unlikely that correlated fluctuations would exist having transverse scales greater than the supergranulation scale of $30,000 \mathrm{~km}$, an estimate that entered above into the first of our three factors. The third factor also depends upon the scale height for gyrofrequency variation $\lambda_{h}(\mathrm{Tu} \&$ Marsch 1997), i.e., the length scale on which the field strength $B$ varies. In the high corona, where $B \sim r^{-2}, \lambda_{h} \approx r / 2$. In the lower corona, super-radial expansion produces smaller $\lambda_{h}$. A typical value in the inner corona may be of order $0.1 R_{\odot}$, i.e., $\lambda_{h}=70,000 \mathrm{~km}$. Consequently, using the largest reasonable $\lambda_{\perp}$ we arrive at the low estimate of the third factor $\lambda_{h} / \lambda_{\perp} \approx 2.3$. We can form a high estimate for the third factor by using a smaller $\lambda_{\perp}$, say $2000 \mathrm{~km}$, the transverse scale of the "furnace" within the network region (Axford \& McKenzie 1997). Then the third factor could be as large as $\approx 35$. However then the high estimate for the first factor should be reduced to about $1.1 \times 10^{3}$.

Putting together the three low estimates, we find that $\epsilon_{\perp} / \epsilon_{\mathrm{cyc}} \approx 13 \times(1 / 33) \times 2.3 \approx 1$. Even in this limit the two effects are comparable. Turning to the high estimate we compute either $7000 \times(1 / 7) \times 2.3 \approx 23,000$, or using the smaller transverse scale, $1100 \times(1 / 7) \times 35 \approx 5500$. In the high estimate regime the transverse cascade heating is many times greater than that due to the cyclotron sweep effect. One concludes, subject to the assumptions made above, that the perpendicular cascade mechanism may be an important factor in the corona, and under certain circumstances may be a dominant factor in heating. It seems unlikely that cascade is negligible. Earlier theoretical treatments (e.g., Tu \& Marsch 1997) examined the cyclotron sweep mechanism for coronal parameters, discarding the direct cascade. On the basis of the estimates above, we suggest that the perpendicular cascade should be evaluated as an alternative mechanism that might drive heating.

To be fair to earlier work, we note that the possibility of a reduced MHD perpendicular cascade had not been men- 
tioned in the open-field-line coronal context. Instead, however, the cascade model that was typically considered was one in which the total energy transfer rate is inversely proportional to $V_{\mathrm{A}}$. This type of model, equivalent to a frequency cascade, appears to be based upon an isotropic cascade model (see, e.g., Kraichnan 1965; Galtier, Politano, \& Pouquet 1997) and does not anticipate strong anisotropy. A decay rate $\propto V_{\mathrm{A}}^{-1}$ is apparently inconsistent with MHD simulations at moderate Reynolds number (Hossain et al. 1995). Thus, the perpendicular cascade is an approach that may motivate a new look at some of these earlier models.

\section{DISSIPATION IN THE SOLAR WIND}

In this section we focus on dissipation in the solar wind. Where appropriate, however, we discuss some implications for coronal dissipation.

\subsection{Anisotropy, Cascades, and Dissipation}

A significant fraction of the energy in solar wind MHDscale fluctuations may reside in quasi-2D regions of the spectrum. In the solar wind typically $b / B_{0} \sim 1$, and the quasi-2D (or reduced MHD) modes occupy a substantial fraction of the entire phase space (Montgomery 1982; Matthaeus et al. 1998; Oughton et al. 1998). The observed spectrum (Matthaeus et al. 1990) has been parameterized as a two-component model, consisting of complementary fractions of two-dimensional and slab modes (respectively, $k_{\|}=0$ and $k_{\perp}=0$ ). Such simplified models have proven useful in transport theory (Tu \& Marsch 1993; Oughton \& Matthaeus 1995) and cosmic-ray scattering theory (Bieber et al. 1994), and emerge naturally in the theory of low Mach number nearly incompressible MHD (Zank \& Matthaeus 1993). A reasonable consensus based upon these studies would be that solar wind fluctuations are consistent with an $80 \%$ two-dimensional and $20 \%$ slab partitioning. This is supported also by direct observational tests using Helios data (Bieber et al. 1996).

Dynamical studies are consistent with a cascade that generates spectra of this type. In a two-component picture, the cascade is dominated by quasi-2D nonlinear couplings (Oughton, Ghosh, \& Matthaeus 1998; Kinney \& McWilliams 1998). A cascade model of this type was applied with some success to the outer solar wind (Zank et al. 1996; Matthaeus et al. 1999b). This model ignores the parallel cascade, assuming, in effect, that parallel transfer is frozen out, as discussed above in connection with Figure 1.

Another type of consistency that one can examine is whether the degree of anisotropy inferred from kinematic studies is consistent with expectations from dynamics. Most dynamical studies of the development of spectral anisotropy are based upon direct numerical simulation (Shebalin et al. 1983; Oughton et al. 1994; Matthaeus et al. 1998). A convenient quantitative measure of spectral anisotropy relative to the mean magnetic field is the anisotropy angle $\theta_{b}$ defined by $\tan \theta_{b}=\bar{k}_{\perp} / \bar{k}_{\|}$, where $\bar{k}_{\perp}$ and $\bar{k}_{\|}$are average perpendicular and parallel wavenumbers computed using the (fluctuating) magnetic energy spectrum as a weight function (see, e.g., Oughton et al. 1994b). For a two-component model with an $80 \%-20 \%$ two-dimensional slab energy apportionment, one can estimate the equivalent anisotropy angle if the two-dimensional and slab spectra are assumed to have the same functional form. One readily finds that the anisotropy angle is $\theta_{b} \approx 70^{\circ}$. Thus, an " $80 \%-20 \%$ " twocomponent model may be described as being, in some sense, equivalent to a more fully populated spectral model with the typical excited Fourier wavevector lying at about $70^{\circ}$ to the mean field direction.

One may also ask whether $\theta_{b}=70^{\circ}$ is consistent with the degree of spectral anisotropy expected on dynamical grounds for the solar wind. Keeping in mind that simulation results are available only at Reynolds numbers much lower than are relevant to the solar wind, (e.g., Matthaeus et al. 1998; Ghosh et al. 1998), we may estimate crudely as follows. Figure 1 of Matthaeus et al. 1998 displays a scaling for $\cos \theta_{\omega}$ versus $b / B$, where $B=\left(b^{2}+B_{0}^{2}\right)^{1 / 2}$ is a measure of the total magnetic field strength and $\theta_{\omega}$ is the anisotropy angle with a weighting function based on the vorticity. If one again assumes that the two-dimensional and slab spectra have the same functional form, then $\theta_{\omega}=\theta_{b}$, and the expected value of anisotropy for solar wind fluctuations can be deduced. One may reasonably adopt an estimate $b / B_{0} \approx 1 / 2$ for the solar wind, or equivalently $b / B \approx 0.44$. From the cited figure we conclude that $\theta_{b} \approx 70^{\circ}$ is expected in the solar wind on the basis of these (low Reynolds number) simulations.

Several lines of argument show consistency between the inferred level of anisotropy of solar wind fluctuations and a cascade dominated by quasi-2D MHD activity. Recent studies of the dissipation range of solar wind fluctuations by Leamon et al. (1998a, 1998b, 1999) provide complementary information in this regard.

1. Observational tests (Leamon et al. 1998b) indicate that the dissipation range consists of an equivalent mixture of approximately 50\% two-dimensional and 50\% slab energy. This contrasts with observations at lower frequency (Bieber et al. 1996), which find the inertial range mixture is about $80 \%$ two-dimensional and $20 \%$ slab. This suggests that the two-dimensional component is dissipated more efficiently. Cyclotron sweep would preferentially attenuate the slab component.

2. Observed cross helicity and magnetic helicity in the dissipation range are consistent with almost equal amounts of cyclotron resonant and noncyclotron-resonant dissipation (Leamon et al. 1998a). Noncyclotron-resonant processes are likely to operate strongly at highly oblique angles.

3. A model based upon linear Vlasov damping and a spectral replenishment approximation produces good agreement with the observed spectral steepening and $1 \mathrm{AU}$ solar wind heating (Leamon et al. 1999). In this model kinetic dissipation maximizes for fluctuations in the $60^{\circ}$ to $70^{\circ}$ range relative to the mean magnetic field.

\subsection{Quasi-2D Current Sheets and Dissipation by Reconnection}

Extrapolating solar wind results to coronal conditions one might expect that perpendicular cascade and quasi-2D fluctuations will play an even more significant role in the dissipation processes (see the discussion surrounding eq. [6]). The question naturally arises as to what MHD dynamical processes might be associated with the formation of these highly oblique structures.

Previous studies have considered the interface between kinetic and MHD effects in the context of linear Vlasov theory, i.e., kinetic Alfvén waves (Leamon et al. 1999). For a cascade picture, it seems appropriate to employ description that is nonlinear. A natural candidate for describing the 
small-scale nonlinear structures produced by an MHD cascade is magnetic reconnection.

It has been argued that many phenomena associated with magnetic reconnection are integral features of twodimensional MHD turbulence (Matthaeus \& Lamkin 1986), including formation of sheets or filaments of electric current density and concentrations of fluid vorticity. The cascade process in two-dimensional MHD probably involves a succession of scale-invariant reconnection processes, with smaller magnetic islands coalescing to form larger poloidal flux structures (Matthaeus \& Montgomery 1980). Meanwhile, energy is cascaded to small scales and dissipated in the associated current sheet regions. The typical current sheet thickness is associated with the dissipation scale (Matthaeus \& Lamkin 1986). The expectation is that a power-law inertial range, dominated by cascade, gives way to a steeper dissipation range spectrum at a wavenumber corresponding to the typical current sheet thickness.

In the solar wind or in the corona, characteristic kinetic phenomena at the dissipative scales should exhibit distinctive signatures. Recent kinetic theory studies have shed considerable light on the nature of kinetic activity associated with reconnection (Biskamp, Schwarz, \& Drake 1997; Shay et al. 1998). In particular, for collisionless reconnection it now appears to be fairly well established that the thickness of the dissipation region, i.e., the current sheet thickness, will be of the order of the ion inertial scale $\rho_{\mathrm{ii}}=c / \omega_{\mathrm{pi}}=$ $V_{\mathrm{A}} / \Omega_{\mathrm{ci}}$. Here $c, \omega_{\mathrm{pi}}, \Omega_{\mathrm{ci}}$ and $V_{\mathrm{A}}$ are the speed of light, the proton plasma frequency, the proton cyclotron frequency, and the Alfvén speed, respectively. Hence for the collisionless case, one would expect that the "breakpoint" that signifies the upper limit of the inertial range should occur typically at the local ion inertial scale.

This suggests observational consequences that are testable using solar wind observations. Previously, Leamon et al. (1998b) employing Wind observations, found that simple cyclotron resonance of parallel-propagating Alfvén waves was not an adequate description of the observed spectral breakpoint. Here we review the Leamon et al. results, but along with the gyrofrequency (Fig. 2) and the parallel cyclotron resonance scale (Fig. 3), we now include the ion inertial scale (Fig. 4). We may ask how these three quantities compare as predictors of the spectral breakpoint frequency.

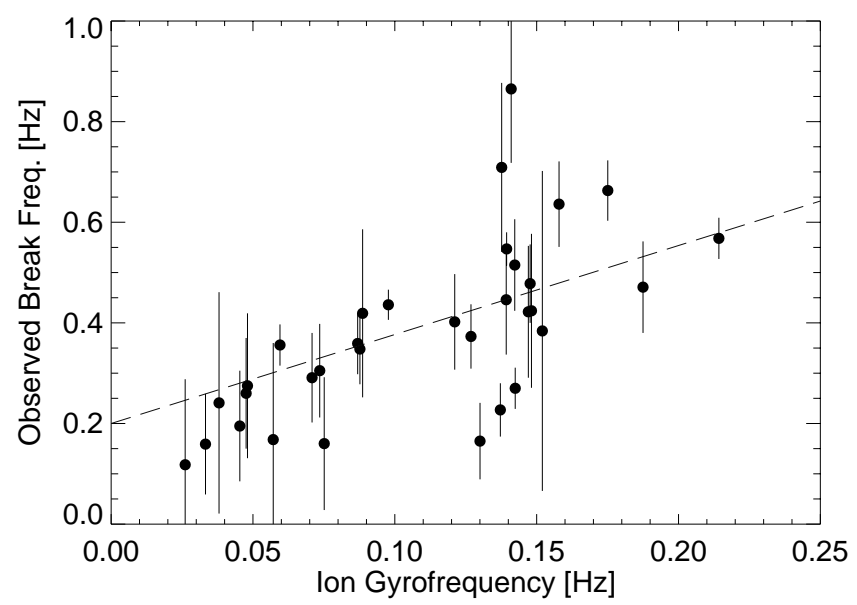

FIG. 2.-Observed breakpoint frequency vs. ion gyrofrequency for 33 Wind intervals (see Leamon et al. 1998b). In this and the following two figures, the dashed line is the linear least-squares best fit. See Table 1 for best-fit parameters.

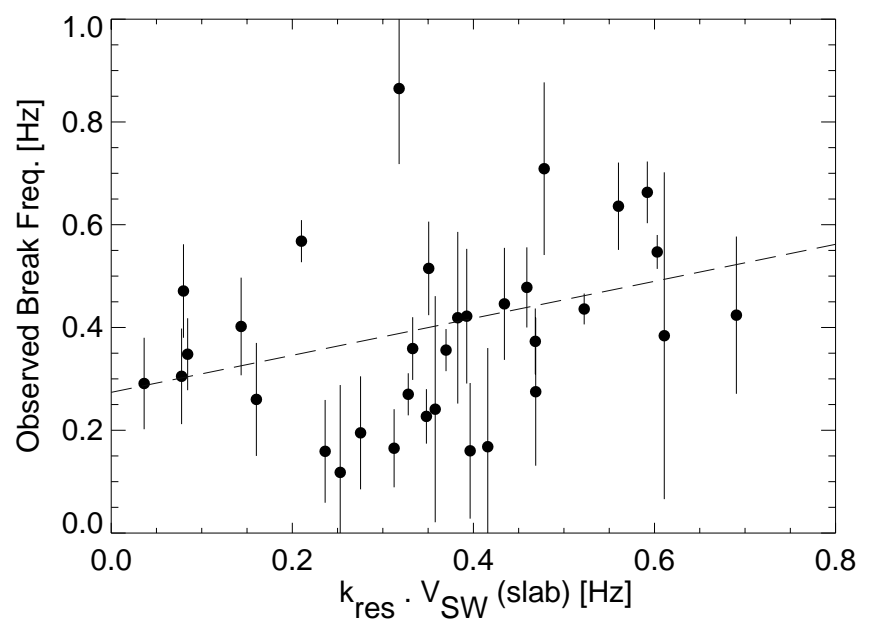

Fig. 3.-Observed breakpoint frequency vs. Doppler-shifted parallel resonant wavenumber for the same data as in Figure 2. See also Table 1.

Figure 2 shows the linear regression of the observed breakpoint $v_{\mathrm{bp}}$ with the observed mean gyrofrequency $\Omega_{\mathrm{ci}}$ for each interval. The parameters for the least-squares fit are given in Table 1, as are those for the best fit constrained to pass through the origin. The error bars on the observed break frequency are calculated by propagation of the errors

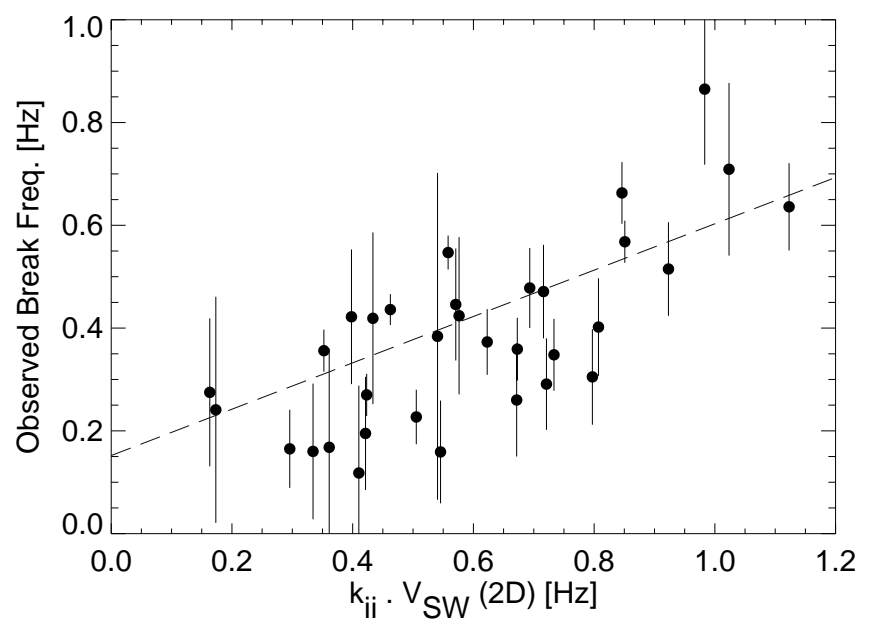

FIG. 4.-Observed breakpoint frequency vs. Doppler-shifted wavenumber of perpendicular (current sheet) structures at the ion inertial scale for the 33 Wind intervals. See also Table 1.

TABLE 1

Best-Fit Parameters for Linear Least SQUARES Fits to THE DATA SHOWN IN Figures 2-4

\begin{tabular}{|c|c|c|c|}
\hline$X$ & $a$ & $b$ & $\chi^{2}$ \\
\hline \multirow[t]{2}{*}{$\Omega_{\mathrm{ci}} \ldots \ldots \ldots \ldots \ldots$} & 0.200 & 1.760 & 2.93 \\
\hline & 0 & 3.190 & 3.88 \\
\hline \multirow[t]{2}{*}{$k_{\mathrm{res}} V_{\mathrm{SW}} \ldots \ldots \ldots$} & 0.274 & 0.360 & 3.61 \\
\hline & 0 & 0.958 & 5.84 \\
\hline \multirow[t]{2}{*}{$k_{\mathrm{ii}} V_{\mathrm{sw}} \ldots \ldots \ldots$} & 0.152 & 0.451 & 2.66 \\
\hline & 0 & 0.686 & 3.07 \\
\hline
\end{tabular}

NoTE. - Fits are of the form $v_{\mathrm{bp}}=a$ $+b(X / 2 \pi)$. Values for both unconstrained fits and fits through the origin are shown. 
in the best-fit inertial and dissipation-range power-law fits to the spectrum, as discussed in Leamon et al. (1998b). For clarity of presentation, errors in the abcissae of Figures 2-4 are neglected.

Now consider the possibility that the damping of slab fluctuations due to cyclotron resonance is responsible for the steeping observed at the breakpoint. Assume that the mean magnetic field is oriented at an angle $\Psi$ (the winding angle) relative to the radial direction. Wavenumbers higher than the resonant wavenumber will show a steepening. Based on the cyclotron resonance condition, the bend-over point in the observed reduced spectrum is expected to occur at radial wavenumber $k_{\text {res }}=\left[\Omega_{\mathrm{ci}} /\left(v_{\mathrm{th}}+V_{\mathrm{A}}\right)\right] \cos \Psi$ in view of the super-Alfvenic flow of the solar wind (the frozen-in flow condition), where $v_{\text {th }}$ is the proton thermal speed. To examine the quality of the parallel resonant wavenumber as a predictor of the observed breakpoint, we seek a linear relation between the spectral breakpoint and $k_{\text {res }}$ (strictly, between $v_{\mathrm{bp}}$ and $k_{\mathrm{res}} V_{\mathrm{SW}}$ ). The results are shown in Figure 3 and Table 1 .

A third case to consider is that current sheets with a purely perpendicular orientation relative to the mean field are the cause of the spectral steepening. This corresponds to strictly two-dimensional turbulence. Assuming a $90^{\circ}$ orientation and a thickness equal to the ion inertial scale, we estimate that the steepening wavenumber is $k_{\mathrm{ii}}=$ $2 \pi \Omega_{\mathrm{ci}} \sin \Psi / V_{\mathrm{A}}=2 \pi v_{\mathrm{bp}} / V_{\mathrm{sw}}$. The data and unconstrained best fit are shown in Figure 4, with the best-fit parameters given in Table 1.

Of the unconstrained linear fits, the best is that for the two-dimensional ion inertial scale, although it is only marginally better than the gyrofrequency fit. The same conclusion holds for the fits through the origin. In each case the parallel resonant wavenumber is a substantially worse predictor of the breakpoint. On this basis alone one would be encouraged in considering kinetic activity near twodimensional current sheets as a possible contributor to solar wind dissipation. However, the picture is clearer when the possibility of oblique wavevectors is included. We can then compare $\rho_{\mathrm{ii}}$ and $k_{\text {res }}$ scaling at various angular orientations.

Suppose there exists an axisymmetric cone of excitations whose wavevectors are at angle $\Theta$ to the mean field and which all have the same magnitude $k_{0}$. As above, let $\boldsymbol{B}_{0}$ form an angle $\Psi$ relative to the (radial) direction of observation. One can show that for a fixed $\Theta$, such a deltafunction distribution of fluctuations has an observed (reduced) energy spectrum with peaks at the maximum projection along the radial direction (see, e.g., the Appendix of Goldstein, Smith, \& Matthaeus 1983). This corresponds to an observed wavenumber of $k_{0} \cos (\Psi-\Theta)$. To generalize the strict two-dimensional case (third case above), suppose that the axisymmetric conical spectrum steepens sharply at wavenumbers corresponding to the current sheet thickness $\rho_{\mathrm{ii}}$. By letting $k_{0}=2 \pi / \rho_{\mathrm{ii}}$, the observed breakpoint frequency then satisfies $v_{\mathrm{bp}} \approx\left(V_{\mathrm{sw}} / 2 \pi\right)\left(\Omega_{\mathrm{ci}} / V_{\mathrm{A}}\right) \cos (\Psi-\Theta)$. Figure 5 (solid curve) shows the results of calculating the best fits to the Wind interval data for each angle of current sheet orientation $\Theta$. The minimum in $\chi^{2}$ for the unconstrained fits is about 1.2 , at $\Theta \approx 65^{\circ}$. This corresponds to current sheets of thickness $\rho_{\mathrm{ii}}(\Theta)$ at rather large angles to the mean field.

In a similar way, the slab resonance condition may be generalized by again assuming a conical distribution, but

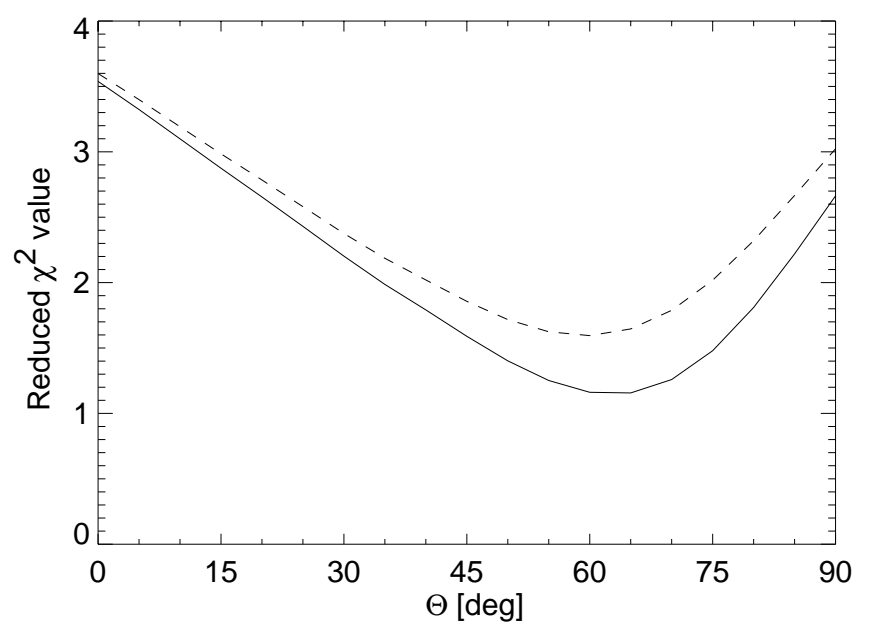

Fig. 5.- Reduced $\chi^{2}$ values, as a function of $\Theta$, for the unconstrained best-fit scalings (see Table 1) of the breakpoint frequency with (1) the ion inertial scale (solid line) and (2) the (cyclotron) resonant wavenumber (dashed line). In each case, the wavevectors at the dissipation scale make an angle $\Theta$ with the mean magnetic field. See text for details. Optimal best fits are obtained for structures that are oblique and at approximately $65^{\circ}$ to the mean field.

now demanding that the parallel resonance condition is satisfied. This requires that $k_{\text {res }} \sim \Omega_{\mathrm{ci}} /\left(v_{\mathrm{th}}+V_{\mathrm{A}}\right)=k_{0} \cos \Theta$. Thus $k_{0}=\Omega_{\mathrm{ci}} /\left[\left(v_{\mathrm{th}}+V_{\mathrm{A}}\right) \cos \Theta\right]$. By applying the same reasoning employed above we conclude that the breakpoint frequency should satisfy $2 \pi v_{\mathrm{bp}} / V_{\mathrm{sw}} \sim\left[\Omega_{\mathrm{ci}} /\left(v_{\mathrm{th}}+V_{\mathrm{A}}\right)\right] \cos$ $(\Psi-\Theta) / \cos \Theta$. Fixing an orientation angle $\Theta$, this provides still another linear relation between breakpoint frequency and physical parameters that can be tested using the Wind breakpoint database. The results are shown in Figure 5 as the dashed curve, allowing a direct comparison of the oblique resonance condition with the oriented ion inertial scale (as predictors of the breakpoint). From the figure we can see that the resonant wavenumber case also reaches a minimum of $\chi^{2}$ at about the same oblique angle. However, the curve lies above the ion inertial scale case for all values of $\Theta$. This result complements the conclusion reached by Leamon et al. 1999 that dissipation occurs at substantially oblique $\left(\approx 70^{\circ}\right)$ angles.

While far from a complete theory of dissipation, these simple examples suggest that the ion inertial scale - and in our interpretation, oblique current sheet activity-play a significant role in dissipation of solar wind turbulence at small scales.

\section{SUMMARY}

We have discussed the role of an anisotropic MHD cascade that results in the kinetic dissipation of fluctuations and the production of thermal energy. For moderate to strong mean magnetic field strengths, and at low plasma beta, the anisotropy of the cascade is expected to be strong. From this, one infers that energy is supplied preferentially to the highly oblique, or quasi-2D, wavevectors. Various indications exist in solar wind observations and theory that this propensity is realized. For coronal conditions, the anisotropy of spectral transfer should be stronger still.

By using direct order-of-magnitude estimates, we have compared the cyclotron sweep mechanism, which acts to dissipate energy at large parallel wavenumbers, and dissipation via a (predominantly) perpendicular cascade. For 
coronal conditions, the cascade mechanism cannot be neglected compared to the sweep mechanism and may be many times larger. This does not invalidate the sweep mechanism as a contributing factor in proton heating, nor does it impact the likelihood that cyclotron sweep may supply energy steadily to minor ions, since this requires a much smaller energy flux.

One is led then to examine the possibility that there may be intrinsically nonlinear channels for dissipation. Here we have suggested that the formation of quasi-2D current sheets is an integral part of the perpendicular cascade process. The current sheets are expected to have a thickness of the order of the ion inertial scale (Shay et al. 1998). This hypothesis was examined in a simple but direct way, employing the same Wind observations of spectral steepening used by Leamon et al. (1998b). We found that the best fit for the cases considered was obtained when the breakpoint is associated with structures of the thickness of the ion inertial scale and oriented obliquely at an angle of approximately $65^{\circ}$ to the mean magnetic field. This is not a full theory for the dissipation process, but it encourages further study beyond the usual cyclotron resonance dissipation mechanism or other processes that occur at the proton gyrofrequency.

Much remains to be addressed in future studies of the interplay between MHD processes and smaller scale kinetic processes. However, based upon the present discussion and the relative success of the cascade picture in the solar wind, we are encouraged to pursue similar models for dissipation and heating in the corona. In view of the expected very low values for the plasma beta, it may be that the significance of quasi-2D current sheets and processes active at the ion inertial scale are even greater in the corona than in the solar wind (Matthaeus et al. 1999a; Shay et al. 1998). This raises intriguing possibilities. One might wonder, for example, whether beams of mean field-aligned suprathermal particles would be accelerated, which would be susceptible to beam instabilities and secondary cyclotron instabilities. Alternatively, magnetosonic modes might be generated by mode conversion near the reconnection regions. These modes are expected to be heavily damped for coronal parameters (Barnes 1979). While the signatures and efficiencies of such secondary nonlinear processes remain to be established, one might hope that the paradigm presented here will be of diagnostic value in discriminating between the competing dissipative processes that are active in the corona and solar wind.

This work is supported by NASA grants NAG 5-6570 and NAG 5-7164, NASA subcontract NAG 5-2848, and NSF grant ATM-9713595 to the Bartol Research Institute.

\section{APPENDIX A}

We may describe the flux $F$ of energy into a specific volume of wavenumber space by summing the transfer function $T$ (see eq. [1]) over a region in $k$-space, denoted by the set $\mathscr{V}$, and defining

$$
F[\mathscr{V} ; t]=\int_{\mathscr{r}} T(\boldsymbol{k}, t) d^{3} k .
$$

In this notation, the isotropic energy transfer function described in eq. (4) is $F_{0}\left(k_{0}, t\right) \equiv F\left[\mathscr{V}_{0}\left(k_{0}\right) ; t\right]$, where $\mathscr{V}_{0}\left(k_{0}\right) \equiv$ $\left\{\boldsymbol{k}:|\boldsymbol{k}|>k_{0}\right\}$. Spectral transfer to higher parallel wave number (see §2.2) may be described by defining a flux $\boldsymbol{F}_{\|}(k)$ such that $F\left[\mathscr{V}_{\|}(k) ; t\right] \approx \epsilon_{\|}$, where $\mathscr{V}_{\|}(k)=\left\{\boldsymbol{k}:\left|k_{\|}\right|>k\right\}$. Similarly, the perpendicular spectral flux $F_{\perp}(k)$ may be defined such that $F\left[\mathscr{V}_{\perp}(k)\right] \approx \epsilon_{\perp}$, where $\mathscr{V}_{\perp}(k)=\left\{\boldsymbol{k}: \mathrm{k}_{\perp}>k\right\}$.

Axford, W. I., \& McKenzie, J. F. 1997, in Cosmic Winds and the Heliosphere (Tucson: Arizona Univ. Press, 31

Barnes, A. 1979, in Solar System Plasma Physics, Vol. I, ed. E. N. Parker, C. F. Kennel, \& L. J. Lanzerotti (Amsterdam: North-Holland), 251

Bieber, J. W., Matthaeus, W. H., Smith, C. W., Wanner, W., Kallenrode, M., \& Wibberenz, G. 1994, ApJ, 420, 294

Bieber, J. W., Wanner, W., \& Matthaeus, W. H. 1996, J. Geophys. Res., 101,2511

Biskamp, D., Schwarz, E., \& Drake, J. F. 1997, Phys. Plasmas, 4, 1002

Chae, J., Schühle, U., \& Lemaire, P. 1998, ApJ, 505, 957

Cranmer, S. R., Field, G. B., \& Kohl, J. L. 1999, ApJ, 518, 937

Cuseri, I., Mullan, D. J., Noci, G., \& Poletto, G. 1999, ApJ, 514, 989

Foukal, P. 1990, Solar Astrophysics (New York: Wiley)

Galtier, S., Politano, H., \& Pouquet, A. 1997, Phys. Rev. Lett., 79, 2807

Ghosh, S., Matthaeus, W. H., Roberts, D. A., \& Goldstein, M. L. 1998, J. Geophys. Res., 103, 23705

Goldstein, M. L., Roberts, D. A., \& Fitch, C. A. 1994, J. Geophys. Res., 99, 11519

Goldstein, M. L., Smith, C. W., \& Matthaeus, W. H. 1983, J. Geophys. Res., 88, 9989

Grall, R. R., Coles, W. A., Klinglesmith, M. T., Breen, A. R., Williams, P. J. S., Markkanen, J., \& Esser, R. 1996, Nature, 379, 429

Grall, R. R., Coles, W. A., Spangler, S. R., Sakurai, T., \& Harmon J. K. 1997, J. Geophys. Res., 102, 263

Habbal, S. R., Esser, R., Guhathakurta, M., \& Fisher, R. R. 1995, Geophys. Rev. Lett., 22, 1465

Hassler, D. M., Rottman, G. J., Shoub, E. C., \& Holzer, T. E. 1990, ApJ, 348, L77

Hollweg, J. V. 1986, J. Geophys. Res., 91, 4111

\section{EFERENCES}

Hollweg, J. V., \& Johnson, W. 1988, J. Geophys. Res., 9547

Hollweg, J. V., \& Turner, J. M. 1978, J. Geophys. Res., 83, 97

Holzer, T. 1977, J. Geophys. Res. 82, 23

Hossain, M., Gray, P. C., Pontius, D. H., Jr., Matthaeus, W. H., \& Oughton, S. 1995, Phys. Fluids, 7, 2886

Isenberg, P. A., \& Hollweg, J. V. 1983, J. Geophys. Res., 88, 3923

Kaghashvili, E. K. 1999, Geophys. Rev. Lett., 26, 1817

Kinney, R., \& McWilliams, J. C. 1998, Phys. Rev., 57, 7111

Kohl, J. L., et al. 1995, Space Sci. Rev., 72, 29 1997, Sol. Phys., 175, 613

Kraichnan, R. H. 1965, Phys. Fluids, 8, 1385

Leamon, R. J., Matthaeus, W. H., Smith, C. W., Wong, H. K. 1998a, ApJ, 507, L181

Leamon, R. J., Smith, C. W., Ness, N. F., Matthaeus, W. H., \& Wong, H. K. 1998b, J. Geophys. Res., 103, 4775

Leamon, R. J., Smith, C. W., Ness, N. F., \& Wong, H. K. 1999, J. Geophys. Res., 104, 22331

Lesieur, M. 1990, Turbulence in Fluids (Dordrecht: Nijhoff)

Matthaeus, W. H., Goldstein M. L., \& Roberts D. Ä. 1990, J. Geophys. Res., 95,20673

Matthaeus, W. H., \& Lamkin, S. L. 1986, Phys. Fluids, 29, 2513

Matthaeus, W. H., \& Montgomery, D. 1980, Ann. NY Acad. Sci., 357, 203

Matthaeus, W. H., Oughton, S., Ghosh, S., \& Hossain, M. 1998, Phys. Rev. Lett., 81, 2056

Matthaeus, W. H., Zank, G. P., Oughton, S., \& Mullan, D. J. 1999a, ApJ, 523, L93

Matthaeus, W. H., Zank, G. P., Smith, C. W., \& Oughton, S. 1999b, Phys. Rev. Lett., 82, 3444

McKenzie, J., Banaszkiewicz, M., \& Axford, W. I. 1995, A\&A, 303, L45 
Montgomery, D. C. 1982, Phys. Scr., 1, 83

Montgomery, D. C., \& Turner, L. 1981, Phys. Fluids, 4, 825

Oughton, S., Ghosh, S., \& Matthaeus, W. H. 1998, Phys. Plasmas, 5, 4235

Oughton, S., \& Matthaeus, W. H. 1995, J. Geophys. Res., 100, 14783

Oughton, S., Priest, E. R., \& Matthaeus, W. H. 1994, J. Fluid Mech., 280, 95

Panchev, S. 1971, Random Functions and Turbulence (New York: Pergammon)

Richardson, J. D., Paularena, K. I., Lazarus, A. J., \& Belcher J. W. 1995, Geophys. Rev. Lett., 22, 325

Schwartz, S. J., Feldman, W. C., \& Gary, S. P. 1981, J. Geophys. Res., 86, 541
Scott, S. L., Coles, W. A. \& Bourgois, G. 1983, A\&A, 123, 207

Shay, M. A., Drake, J. F., Denton, R. E., \& Biskamp D. 1998, J. Geophys. Res., 103, 9165

Shebalin, J. V., Matthaeus, W. H., \& Montgomery, D. 1983, J. Plasma Phys., 29, 525

Tu, C.-Y., \& Marsch, E. 1993, J. Geophys. Res., 98, 1257 1997, Sol. Phys., 171, 363

Tu, C.-Y., Pu, Z.-Y., \& Wei, F.-S. 1984, J. Geophys. Res., 89, 9695

Zank, G. P., \& Matthaeus, W. H. 1992, J. Plasma Phys., 48, 85

.1993, Phys. Fluids, A5, 257

Zank, G. P., Matthaeus, W. H., \& Smith, C. W. 1996, J. Geophys. Res., 101, 17093 